\title{
Development and characterization of phytosterol nanoemulsions and self-microemulsifying drug delivery systems
}

YUAN Chuanxun ${ }^{1}$,ZHANG Xueru ${ }^{1}$, JIN Risheng ${ }^{1 *}$

${ }^{1}$ Engineering Research Center of Bioprocess Ministry of Education, Hefei University of Technology, Hefei,

Anhui, China.

*jinrisheng@hfut.edu.cn

\section{Abstract}

The aim of this study is to develop a self microemulsion drug delivery system for phytosterols to improve the solubility and bioavailability. The results showed that the formulation of phytosterol self- microemulsion is: lemon essential oil in oil phase, polyoxyethylene hydrogenated castor oil 40 and Tween 60 in emulsifier, polyethylene glycol 400 in co-emulsifier, $\mathrm{Km}=7: 3, \mathrm{Kp}=3: 1$, $\mathrm{Ke}=50 \%$. The drug loading of phytosterol self-microemulsion prepared by this method was $87.22 \mathrm{mg} / \mathrm{g}$, encapsulation efficiency was $89.65 \%$, particle size was $48.85 \mathrm{~nm}$, potential was $-12.863 \mathrm{mV}$. In vitro release experiment showed that the release of phytosterols in microemulsion was more than $90 \%$, and the release curve was in accordance with the first-order kinetics equation. The pharmacokinetic analysis of PSSM synthesized by this method shows that PSSM can increase the bioavailability of PS more than three times, so it is necessary to do more in-depth research on the self-microemulsion delivery system of phytosterols.

\section{Introduction}

Phytosterols(PS) are triterpenes, an important component of plant cells, and are commonly found in seeds, vegetable oils and grains. Common in nature are $\beta$-sitosterol, campesterol, stigmasterol and ergosterol [1].It has the physiological functions of lowering blood lipid [2, 3] , lowering cholesterol [4-6], preventing cardiovascular disease [7],anti-cancer[8], anti-oxidation, scavenging free radicals[9, 10], enhancing immunity[11], anti-inflammatory [12, 13] and so on.

Especially in reducing blood lipids, PS and cholesterol have similar molecular structure, which can competitively inhibit the absorption of cholesterol in the human intestinal tract, thereby reducing blood lipids [14]. Studies have shown that taking $2 \mathrm{~g}$ of PS a day can reduce low density lipoprotein(LDL)by $8.8 \%[15]$.Like unsaturated fatty acids and cholesterol, PS is easy to oxidize and can produce various phytosterol oxidation products (POPs), such as hydroxyl groups, epoxy resins, ketones and triol derivatives, especially when heated or stored for a long time[16].PSs are insoluble in water and slightly soluble in oil, which limits their application in the pharmaceutical and health products industries. Self-microemulsion drug delivery system (SMEDDS) improves the bioavailability of drugs by improving their solubility, lymphatic absorption and intestinal permeability[17].In addition, the microemulsion has good stability[18], which can reduce the oxidation deterioration caused by the storage of PS. In this study, phytosterol self-microemulsion (PSSM) was prepared, the release characteristics of phytosterol in vitro and the pharmacokinetic analysis were investigated by reverse dialysis, which provided a theoretical basi for the study of PSSM.

\section{Materials and methods}

\section{Materials}


Phytosterol with purity of $98 \%$ was purchased from Xi'an Ruiying Biotechnology Co., Ltd. (Xi'an, China). Polyoxyethylene hydrogenated castor oil 40(HCO-40), tween 20, tween 60, tween 80, isopropyl myristate(IPM), 1,2-propanediol, polyethylene glycol 400 (PEG400) and polyoxyethylene castor oil (EL) were obtained from Shandong Yousuo Chemical Technology Co., Ltd. (Shandong, China). Lemon essential oil(LEO), linoleic acid(LA), oleic acid(OA) and ethyl oleate(EO) were purchased from Shandong Baihong New Materials Co., Ltd. Methanol (chromatographic purity, Tedia Company); the other reagents are analytical reagents. The experimental water is distilled water.

\section{Oil-water partition coefficiency}

$10 \mathrm{ml} \mathrm{n}$-octanol was measured and placed in three conical bottles. Distilled water, $0.1 \mathrm{~mol} / \mathrm{HCL}$ and pH6.8 phosphate buffer(pH6.8 PBS) were added to each bottle. The two phases were saturated and separated overnight. The saturated n-octanol phase and three medium water phases were obtained[19]. The drug was dissolved in n-octanol until saturated.The supernatant was centrifuged (5000r/min, 10min) and the original solution was obtained. $2 \mathrm{ml}$ of original solution and $2 \mathrm{ml}$ of the above three saturated water were mixed and kept for the night.The drug concentrations in original solution and three saturated water phases were determined by HPLC. The formula of oil-water partition coefficient $(\mathrm{P})$ was as follows:

$$
\mathrm{P}=\left(C_{0}-C_{\mathrm{w}}\right) / C_{\mathrm{w}}
$$

where $\mathrm{C}_{0}$ represents the drug concentration in original solution; $\mathrm{C}_{\mathrm{w}}$ is the drug concentration in the aqueous phase after equilibrium.

\section{Solubility studies}

PS was added to different oil phases and emulsifiers to saturate, shaking for $48 \mathrm{~h}\left(37{ }^{\circ} \mathrm{C} 100 \mathrm{r} /\right.$ $\mathrm{min})$, static equilibrium for $24 \mathrm{~h}$, centrifugation $(5000 \mathrm{r} / \mathrm{min}, 10 \mathrm{~min})$. The upper liquid was diluted 10 times and detected by HPLC.

\section{Pseudo-ternary Phase Diagram}

Oil phase and emulsifier with high solubility were selected to combine with co-emulsifier. The self-microemulsion system was formed by mixing the oil phase with its mass ratio of $9: 1,8: 2,7: 3$, $6: 4,5: 5,4: 6,3: 7,2: 8,1: 9$. With water drop method, visual observation and laser pen assistance, the critical point of clarification and path formation was recorded. The percentage of oil phase, emulsifier phase and water phase in the whole system was calculated, and pseudo-ternary phase diagram was drawn[20].

\section{Preparation of PSSM}

Adding 4.00g LEO, 2.40g HCO-40, 1.03g Tween 60, 1.14g PEG400 and 2.00g PS, shaking in shaking flask ( $37 \mathrm{C}, 100 \mathrm{r} / \mathrm{min}$ ) for 48 hours, standing for 24 hours, removing undissolved PS, and obtaining PSSM[21].

\section{Particle size and zeta-potential}

The prepared SM was diluted 50 times with distilled water at $37^{\circ} \mathrm{C}$. The particle size, polydispersity index (PDI) and Zeta potential of the microemulsion were measured. All samples 
were balanced for $120 \mathrm{~s}$ at $25{ }^{\circ} \mathrm{C}$ before measurement and each sample was measured three times in parallel with Zetasizer Nano S90 High Sensitivity Nano Particle Size Analyzer (UK Malvern Instruments Co., Ltd.)[22].

\section{Drug loading and entrapment efficiency}

SM $25.00 \mathrm{mg}$ in $10 \mathrm{ml}$ volume bottle was shaken with methanol until calibration,ultrasound was performed for 10 minutes, concentration was determined by HPLC and recorded as W1[23] $.100 .00 \mathrm{mg} \mathrm{SM}$ in $5 \mathrm{ml}$ volume bottle was shaken with distilled water to scale. After 12 hours of dialysis(MWCO $500 \mathrm{Da}$ ), the SM was diluted with methanol for 10 times and demulsified by ultrasound for 30 minutes then W2 was determined by HPLC. The formula for determination of drug loading(DL) and entrapment efficiency (EE) are as follow[24]:

$$
\mathrm{DL}(\mathrm{mg} / \mathrm{g})=W_{1} \times 1000 \quad \text { (2) } \quad \mathrm{EE}(\%)=\frac{W_{2}}{W_{1}} \times 100
$$

where $W_{1}$ is PS content in self microemulsion, and $W_{2}$ is PS content in microemulsion.

\section{Morphology observation}

The morphology of PSSM prepared under optimum conditions was observed by transmission electron microscopy (TEM). Test conditions: $200 \mathrm{kV}$ acceleration voltage, No. 1 condenser aperture.Take $0.1 \mathrm{~g} \mathrm{SM}$, dilute it 50 times with distilled water at $37^{\circ} \mathrm{C}$, put a drop on copper, absorb excess liquid with filter paper, dye it with $2 \%$ phosphotungstic acid solution and dry it, and observe it under JEM-2100F Field Emission Transmission Electron Microscope (Japan)[12].

\section{Stability of PSSM}

Three samples of the same PSSM were stored at 4, 25 and 40 degrees for 45 days. The particle size changes were detected every 5 days and the precipitation degree of the drug during storage was observed.

\section{In vitro release study}

Study on in vitro release of PSSM and phytosterol raw materials(PSRM) by reverse dialysis, the dilution media were distilled water, $\mathrm{pH}$ 6.8PBS and $0.1 \mathrm{~mol} / \mathrm{L} \mathrm{HCL}$ solution, the parameters of magnetic stirring water bath were $37^{\circ} \mathrm{C}, 50 \mathrm{r} / \mathrm{min}$. Ten dialysis bags (MWCO 12,000-14,000 Da) were added with $5 \mathrm{ml}$ dilution medium, balanced for $12 \mathrm{~h}, 4.00 \mathrm{~g} \mathrm{SM}$ in $900 \mathrm{ml}$ dilution medium with $1 \%$ Tween 80 . Take out a dialysis bag respectively at appropriate intervals and $5 \mathrm{ml}$ of the same release medium was supplemented[25]. The drug concentration in dialysis bags was determined by HPLC, and the drug release curve was drawn.

\section{Pharmacokinetic study}

Twelve male SPF Sprague-Dawley rats $(240+10 \mathrm{~g})$ were fed adaptively for 7 days. The temperature and humidity of the animal room were $25 \pm 2^{\circ} \mathrm{C}, 55 \pm 5 \%$ and $12 / 12 \mathrm{~h}$ light-dark cycles. All animals used in the study were treated in strict accordance with the laboratory animal care and use guidelines of the National Institutes of Health. All procedures were approved by the Animal Nursing Assessment Committee of Hefei University of Technology, China.

SD rats were randomly divided into PSSM and PSRM groups, two groups were fed with the same amount of PS . Six rats in each group were fed $10 \mathrm{mg} / \mathrm{g}$ (PS content is $87.22 \mathrm{mg} / \mathrm{g}$ ), fasting 24 
hours before administration and drinking water freely. At the appropriate time point, about $0.3 \mathrm{~mL}$ of blood was collected from the posterior orbital venous plexus of rats and placed in a heparinized centrifugal tube. The plasma was separated by centrifugation at $6000 \mathrm{rpm}$ for 10 minutes and stored at $-80^{\circ} \mathrm{C}[26]$. The content of PS in plasma was determined by HPLC, and the data of plasma concentration were processed by WinNonlin 5.2 software[27]. The pharmacokinetic parameters are: area under curve (AUC); peak concentration (Cmax); time peak concentration (tmax) and relative bioavailability (F\%). The formulas are as follows [28, 29]:

$$
\mathrm{F} \%=\frac{A U C_{T} / \text { Dose }_{T}}{A U C_{R} / \text { Dose }_{R}} \times 100 \%
$$

where $T$ refers to the test preparation, $R$ is the reference preparation.

\section{Conditions of HPLC}

Chromatographic column: Eclipse plus C18 (Agilent, $4.6 \times 250 \mathrm{~mm} / 5 \mu \mathrm{m}$ ); mobile phase: methanol: water $=99: 1$; flow rate: $1.0 \mathrm{~mL} / \mathrm{min}$; detection wavelength: $210 \mathrm{~nm}$; column temperature: $30^{\circ} \mathrm{C}$; injection volume: $10 \mu \mathrm{L}$.

\section{Results and discussion}

\section{Oil-water partition coefficiency}

The drug distribution coefficient $\mathrm{P}$ in n-octanol-water system is a physical constant that simulates the lipophilicity or hydrophobicity of drugs in vivo, and is used to predict the absorption of drugs in the intestine[30]. When $0<\log \mathrm{P}<3$, the drug can be absorbed by gastrointestinal tract, when $\log \mathrm{P}<0$, the drug is hydrophilic; when $\log \mathrm{P}>3$, the drug is lipophilic, but neither of them is easily absorbed by gastrointestinal tract.It is generally believed that $\log P$ between 2 and 3 is easily absorbed, while drugs with $\log \mathrm{P}<2$ are not easily absorbed[31, 32]. Table 1 shows that the $\log \mathrm{P}$ of phytosterols in the three media is less than 2 , indicating that phytosterols are difficult to be absorbed by intestine. Therefore, it is necessary to improve the dosage forms of phytosterols.

Table 1 Apparent partition coefficient of phytosterol in different solvents

\begin{tabular}{llll}
\hline & distilled water & $0.1 \mathrm{~mol} / \mathrm{L}$ HCL & $\mathrm{pH6.8PBS}$ \\
\hline $\mathrm{P}$ & 1.762 & 41.69 & 12.88 \\
\hline $\log \mathrm{P}$ & 0.246 & 1.62 & 1.11 \\
\hline
\end{tabular}

\section{Solubility studies}

The oil phase selected by SMEDDS generally has high solubility for drugs[33]. The solubilities of LEO, LA, EO, OA and IPM were determined. As shown in Fig.1 , the solubilities of different oils to phytosterols were as follows: LEO $>$ OA $>$ IPM $>$ LA $>$ EO.Surfactants have certain toxicity. Generally, polyoxyethylene vegetable oil is less toxic than single-chain surfactants and lipid surfactants are less toxic than ethers. Non-ionic surfactants are preferred for oral SM.Commonly used nonionic surfactants are tween, polyoxyethylene, polyethylene glycerol, Sipan and natural lecithin[34-36].In this experiment, Tween 20, Tween 60, Tween 80, HCO-40 and EL were 
selected to determine the solubility of PS. The results showed that Tween $60>$ Tween $80>$ polyoxyethylene castor oil > polyoxyethylene hydrogenated castor oil > Tween 20 (Fig.2). So LEO and tween 60 were selected as the oil phase and emulsifier of PSSM. After many preliminary experiments, it was found that the particle size of the prepared microemulsion was larger than 300 $\mathrm{nm}$.But after adding HCO-40 in microemulsion, the particle size of microemulsion decreases obviously. The reason may be that HCO-40 and Tween 60 have complementary hydrophilic group conformations and play a synergistic role in compound emulsifiers (this part may be further discussed in future research). In summary, the PSSM oil phase is lemon essential oil, and the emulsifiers are compounds of HCO-40 and Tween 60 .

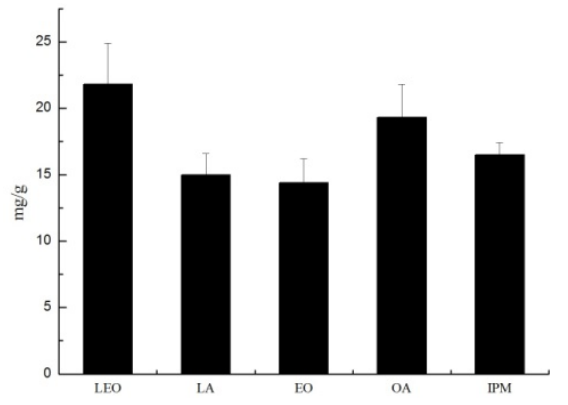

Fig 1. Solubility of phytosterols in different oil phases

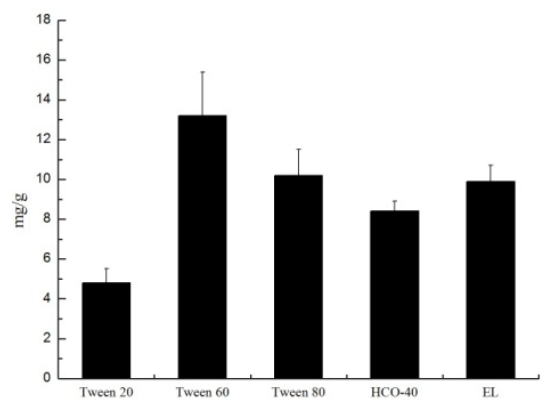

Fig 2. Solubility of phytosterols in different emulsifiers

\section{Pseudo-ternary phase diagram}

The larger the area of microemulsion in pseudo-ternary phase diagrams, the easier the system to form microemulsion and the more stable it is[37, 38].In this part of the experiment, the single factor variable method was used to select the appropriate co-emulsifier and its content with the area of microemulsion as the index.2.00g LEO, 1.50g HCO-40 and 1.50g Tween 60 were added with $1.00 \mathrm{~g}$ absolute ethanol, 1,2-propanediol and PEG400 to determine the appropriate co-emulsifier, or $12.00 \mathrm{~g}, 9.00 \mathrm{~g}, 6.00 \mathrm{~g}, 3.00 \mathrm{~g}, 1.50 \mathrm{~g}, 1.00 \mathrm{~g}, 0.75 \mathrm{~g}$ co-emulsifier , that is, the ratio of emulsifier to co-emulsifier $(\mathrm{Kp})=1: 4,1: 3,1: 1,2: 1,3: 1,4: 1$, respectively, to determine the appropriate Kp.As can be seen from Figs.3 and Table 2, when PEG400 is used as co-emulsifier and $\mathrm{Kp}=3: 1$, the proportion of microemulsion area in pseudo-ternary phase diagram is the largest. Therefore, PEG400 was chosen as emulsifier and $\mathrm{Kp}=3: 1$.

(A)

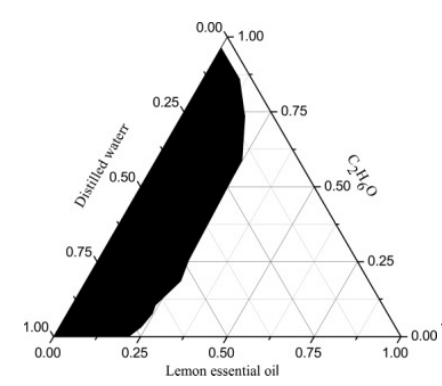

(B)

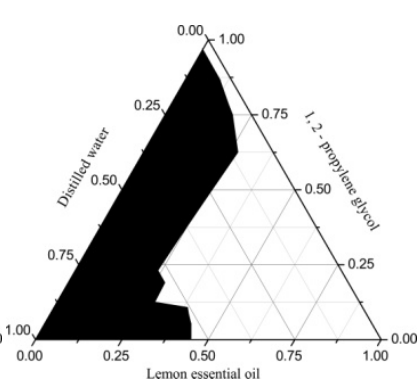

(C)

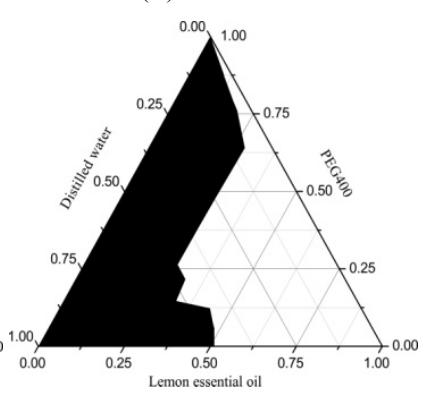

Fig 3. Pseudoternary phase diagrams were drawn with different co-emulsifiers.(A) Anhydrous ethanol; (B) 1, 2 - propylene glycol ;(C) PEG400 .( The shaded part of the pseudo-ternary phase diagram is the microemulsion region )

Table 2 The proportion of microemulsionb in pseudo ternary phase diagrams with different $\mathrm{Km}$

\begin{tabular}{llllllll}
\hline $\mathrm{Kp}$ & $1: 4$ & $1: 3$ & $1: 2$ & $1: 1$ & $2: 1$ & $3: 1$ & $4: 1$ \\
\hline Microemulsion & $49.31 \%$ & $55.72 \%$ & $53.88 \%$ & $51.12 \%$ & $56.04 \%$ & $58.24 \%$ & $51.97 \%$ \\
\hline
\end{tabular}


ratio $^{\text {a }}$

Table 2 are the percentages of shadow area in the corresponding pseudo-ternary phase diagram calculated by AutoCAD 2012 software in the total pseudo-ternary phase diagram area.

\section{Particle size and zeta-potential}

SMEDDS is a thermodynamic and dynamic stabilization system which can form particle size 10-100nm after emulsification. Particle size distribution is a standard for measuring the formation of microemulsion[39, 40]. Polydispersity index (PDI) is an indicator of particle size distribution. It should generally be between 0 and 0.3 , and should not exceed 0.7 . The smaller the value, the more concentrated the particle size distribution and the more uniform.Zeta potential is one of the indexes to determine the physical stability of microemulsion, and it is an index to measure the intensity of mutual exclusion or attraction between particles. The greater the zeta potential, the more stable the system is. The particle size of microemulsion is related to the speed and degree of drug release. The smaller the particle size, the larger the interface area, and the easier the diffusion to gastrointestinal juice, the more complete the drug release[41].

2.00g LEO, 1.00G PEG400, 3.00g emulsifiers were used, in which the mass ratio of HCO-40 to Tween60 (Km) was 1:9, 2:8, 3:7, 4:6, 5:5, 6:4, 7:3, 8:2, 9:1, diluted 50 times with distilled water. The optimum $\mathrm{Km}$ was determined by single factor variable method with particle size, PDI and zeta potential as indexes. Similarly, take $2.00 \mathrm{~g}$ LEO and $1.00 \mathrm{~g}$ PEG400, respectively, and take the emulsifier to the total system mass ratio (Ke) of $20 \%, 30 \%, 40 \%, 50 \%, 60 \%, 70 \%, 80 \%, \mathrm{Km}=$ 5:5, to study the best Ke. From Fig. 4 and 5, the best $\mathrm{Km}=7: 3$ and the best $\mathrm{Ke}=50 \%$.In conclusion, the formulation of PSSM is: LEO in oil phase, HCO-40 and Tween 60 in emulsifier, PEG400 in co-emulsifier, $\mathrm{Km}=7: 3, \mathrm{Kp}=3: 1, \mathrm{Ke}=50 \%$.

(A)

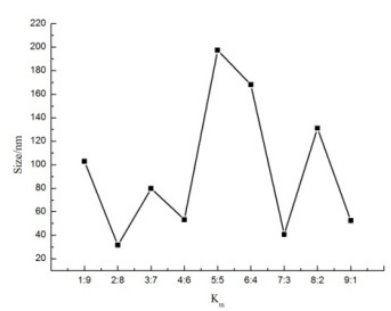

(B)

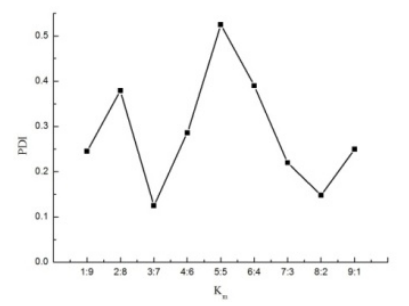

(C)

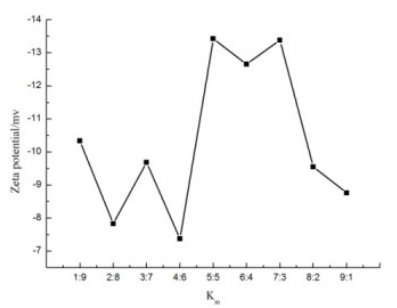

Fig 4. The relation between $\mathrm{Km}$ and particle size, PDI, potential. (A) particle size ;(B) PDI ;(C) potential.

(A)

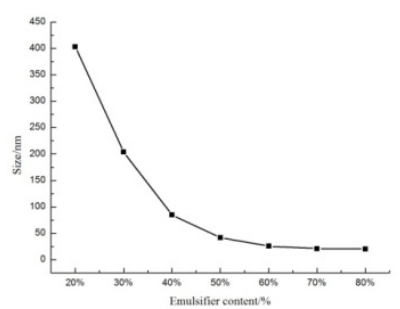

(B)

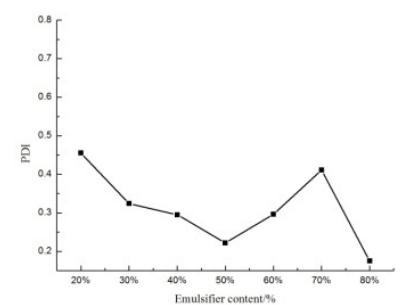

(C)

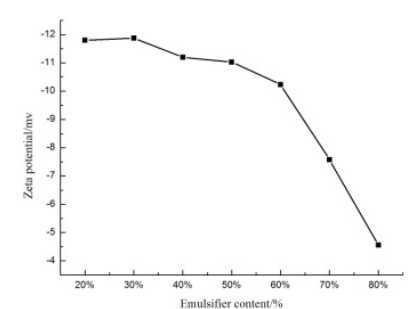

Fig 5. The relation between Ke and particle size, PDI, potential .(A) particle size ;(B) PDI ;(C) potential.

\section{Evaluation of PSSM}

\section{Drug loading and encapsulation rate}

Drug loading refers to the ratio of the drug content in the system to the total weight of the system. 
Encapsulation ratio refers to the ratio of the total drug quantity encapsulated in the system to the total drug input in the system[42]. In this part, the drug loading, encapsulation efficiency, particle size, PDI and potential of the prepared PSSM were tested. The results were shown in table 3, and the particle size and potential distribution were shown in Fig.6 and 7.

Table 3 The drug loading, encapsulation efficiency, particle size, PDI and potential of the PSSM

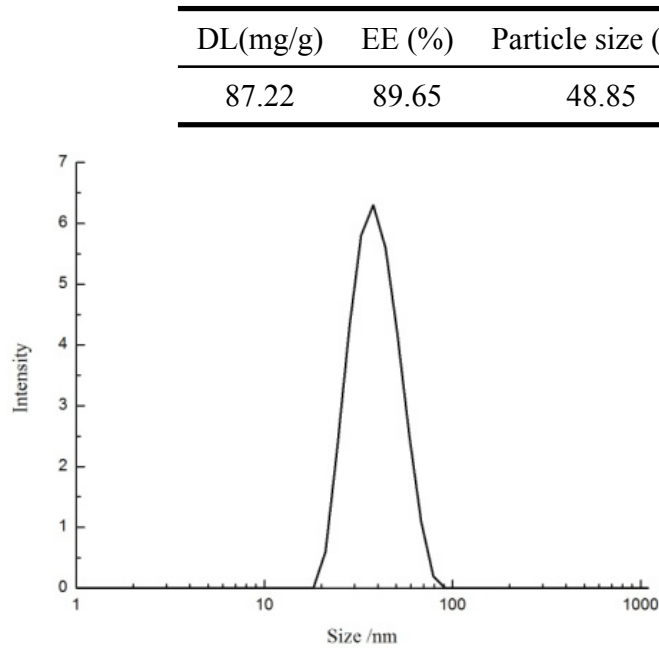

Fig 6. Particle size distribution

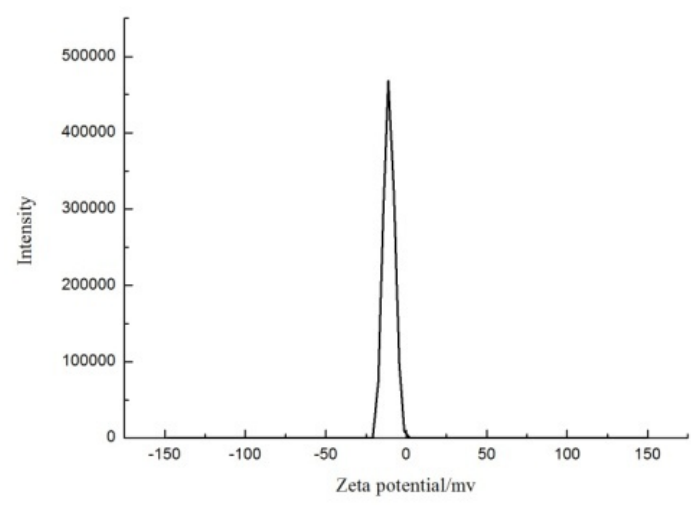

Fig 7. Zeta potential distribution diagram

\section{Morphology observation}

The PSSM was diluted 50 times with distilled water and observed by TEM. In Fig. 8(A) and (B) are microemulsions with different magnifications enlarged under the same field of vision. From Fig. 8(A), we can see that the particle size of microemulsions is relatively uniform and evenly distributed in the whole field of vision. as can be seen from Fig.8(B), the morphology of the microemulsion is spherical, and there is little adhesion between microemulsion particles. The particle size is about $48 \mathrm{~nm}$, which is consistent with the particle size measured by Zeta potentiometer.

(A)

(B)

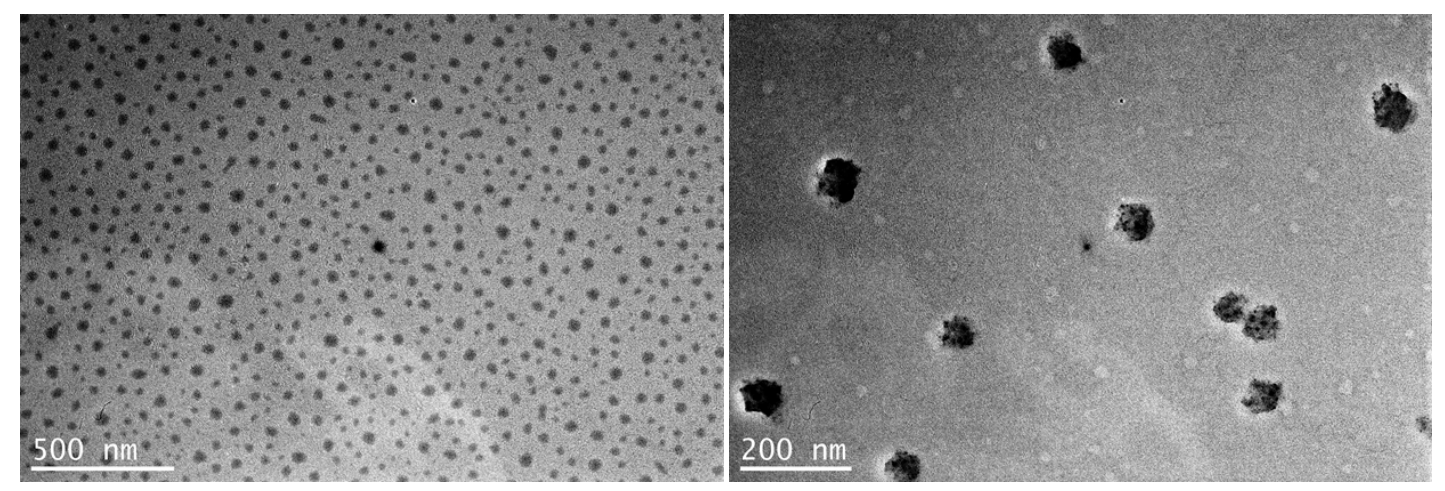

Fig 8. TEM micrograph of the PSSM.(A) TEM micrograph of the PSSM at magnitude 20,000×. (B) TEM micrograph of the PSSM at magnitude 50,000×.

\section{Stability of PSSM}

The particle size change of PSSM during storage in different environments is shown in Fig. 9. It can be seen that the particle size of PSSM varies significantly at $4^{\circ} \mathrm{C}$ and $40^{\circ} \mathrm{C}$ than at $25^{\circ} \mathrm{C}$, the particle size change range is relatively small. As can be seen from Fig. 10, PSSM did not 
precipitate in different environments for 45 days. In conclusion, PSSM has good storage stability.

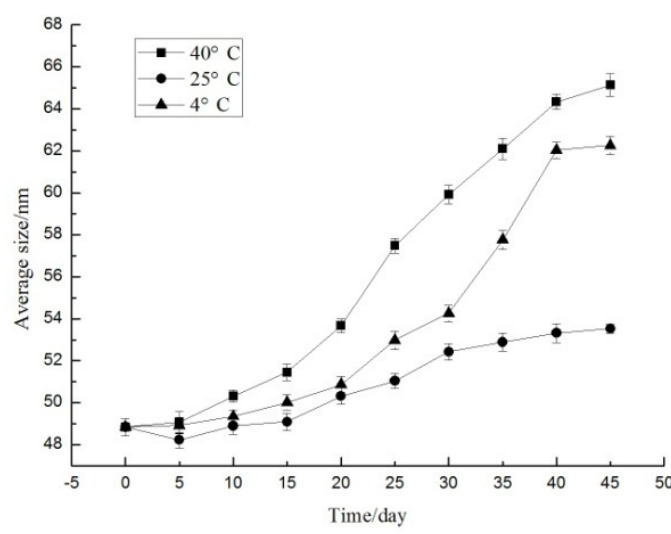

Fig 9. Particle size change during storage of PSSM

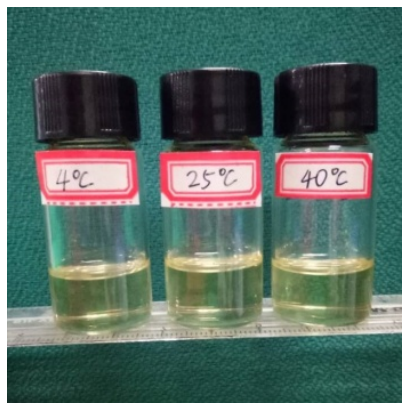

Fig10. PSSM after 45 days storage

\section{In vitro release study}

In vitro drug release is an important index for evaluating the drug delivery system of granules. The drug release behavior and mechanism in vivo can be predicted by in vitro drug release experiments, and the structure of granules can be understood. At present, dialysis, reverse dialysis, ultrafiltration and Franz diffusion cell methods are commonly used to evaluate the in vitro release of SMEDDS[43, 44]. The similarity of release curves was compared by similarity factor (f2) method with DDSolver software and the drug release equation was fitted[45]. Fig.11 shows that PSSM release completely within $3 \mathrm{~h}$ in three dilution media and there is no significant difference in drug release. Table 4 shows that $50<\mathrm{F} 2<100$, indicating the three release curves are similar to each other [46] . Fig.12 shows that PSRM almost completely released in about an hour, and the release rate was higher than that of PSSM.The results show that the release mechanism of PSSM conforms to the first-order kinetics equation (Table 5).

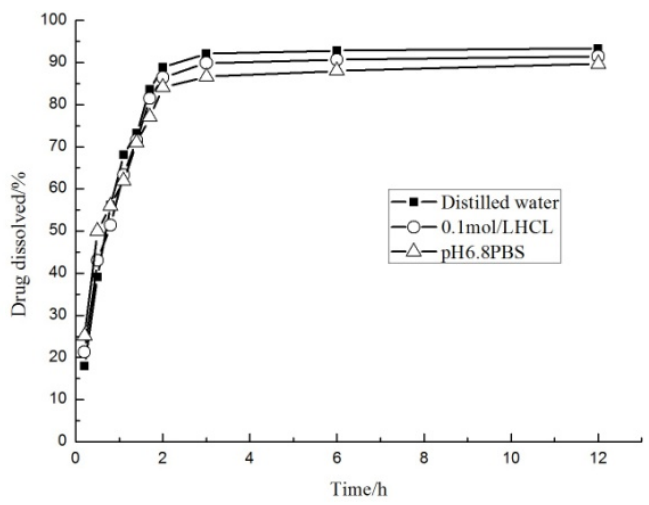

Fig 11. Release curve of PSSM in different dilution media pH 6.8 PBS

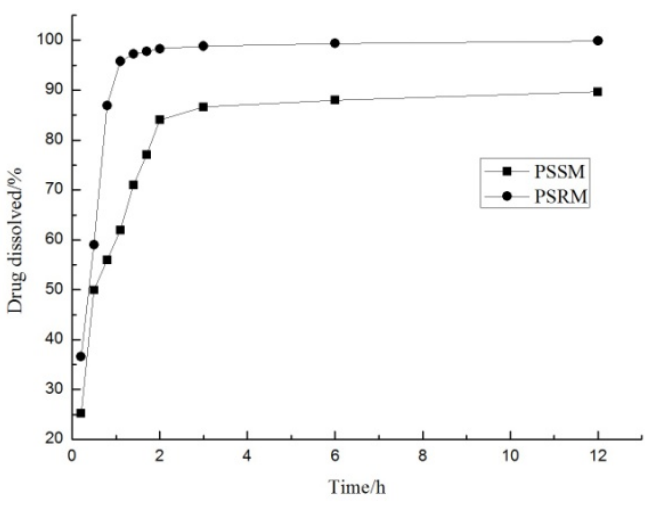

Fig 12.Release curve of PSSM and PSRM in

Table 4 In-vitro Dissolution Profile Comparison in different diluents

\begin{tabular}{llll}
\hline Statistics & HCL - H2O & H2O-PBS & HCL-PBS \\
\hline Observed f2 & 74.132 & 61.234 & 71.162 \\
Number of bootstrap & 5000 & 5000 & 5000 \\
Bootstrap mean & 74.132 & 61.234 & 71.162 \\
Bootstrap median & 74.132 & 61.234 & 71.162 \\
$5 \%$ percentile & 74.132 & 61.234 & 71.162 \\
\hline
\end{tabular}




\begin{tabular}{llll}
\hline 95\% percentile & 74.132 & 61.234 & 71.162 \\
Skewness & -1.000 & -1.000 & -1.000 \\
Kurtosis & -2.001 & -2.001 & -2.001 \\
Is 5\% percentile $\geq 50$ & Yes & Yes & Yes \\
Similarity of R and T & Accept & Accept & Accept \\
\hline
\end{tabular}

Table 5 Regression equation and data fitting of PSSM release in pH 6.8 PBS

\begin{tabular}{lll}
\hline Dissolution Data Modeling & Equation & Rsqr \\
\hline Zero-order with F0 & $\mathrm{F}=58.524+3.628 * \mathrm{t}$ & 0.3973 \\
First-order with Fmax & $\mathrm{F}=87.657 *[1-\operatorname{Exp}(-1.340 * \mathrm{t})]$ & 0.9665 \\
Higuchi with F0 & $\mathrm{F}=42.379+18.135 * \mathrm{t}^{\wedge} 0.5$ & 0.6121 \\
Hixson-Crowell with Tlag & $\mathrm{F}=100 * 【 1-[1-0.098 *(\mathrm{t}+1.570)]^{\wedge} 3 】$ & 0.7686 \\
Korsmeyer-Peppas with F0 & $\mathrm{F}=-71592.330+71653.858 * \mathrm{t}^{\wedge} 0.000$ & 0.8695 \\
\hline
\end{tabular}

\section{Pharmacokinetic study}

As shown in Fig. 13 , after taking the same dose of PSSM and PSAM orally, the concentration of PS in PSSM group remained at 0.79-2.87 ug/ml within 24 hours, which was significantly higher than that in PSAM group (0.12-1.36 ug/ml). The Cmax value of PSSM group was $2.89 \pm 0.20$ at $\operatorname{Tmax}=2.63 \pm 0.36 \mathrm{~h}$, which was 1.55 times higher than that of PSAM group at Tmax $=1.70 \pm 0.12$ $\mathrm{h}$ (Table 6). The CL of PSSM group $(3.94 \pm 0.87 \mathrm{ug} / \mathrm{h})$ was significantly lower than that of PSAM group $(15.35 \pm 1.41 \mathrm{ug} / \mathrm{h})$. The bioavailability of PSSM group was $389.68 \%$ compared with that of PSAM group. Therefore, PSSM can significantly improve the bioavailability of PS.

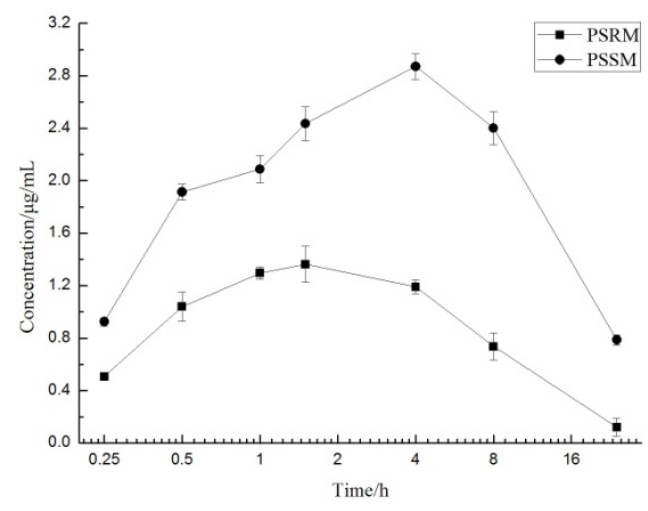

Fig 13. Mean plasma concentration-time profiles of PS in rats

Table 6 Pharmacokinetic parameters of PSSM and PSAM

\begin{tabular}{cccccc}
\hline Formulation & $\mathrm{AUC}(\mu \mathrm{g} / \mathrm{mL}) * \mathrm{~h}$ & $\mathrm{Tmax}(\mathrm{h})$ & $\mathrm{Cmax}(\mu \mathrm{g} / \mathrm{mL})$ & $\mathrm{CL}(\mu \mathrm{g} / \mathrm{h})$ & $\mathrm{F}(\%)$ \\
\hline PSSM & $59.66 \pm 12.24$ & $2.63 \pm 0.36$ & $2.89 \pm 0.20$ & $3.94 \pm 0.87$ & \multirow{2}{*}{ PSAM } \\
\hline
\end{tabular}

\section{Conclusion}

In this study, by measuring the oil and water distribution coefficient of phytosterol, we know that phytosterol is not easily absorbed by human intestinal tract, so it is necessary to carry out studies on microemulsion preparation of phytosterol. In the process of formulation optimization of PSSM, solubility test, drawing of pseudo ternary phase diagram and particle size and potential test were 
carried out, and the preparation prescription of PSSM was determined as follows: lemon essential oil in oil phase, polyoxyethylene hydrogenated castor oil 40 and Tween 60 in emulsifier, polyethylene glycol 400 in co-emulsifier, $\mathrm{Km}=7: 3, \mathrm{Kp}=3: 1, \mathrm{Ke}=50 \%$. The pharmacokinetic analysis of PSSM synthesized by this method shows that PSSM can increase the bioavailability of PS more than three times, so it is necessary to do more in-depth research on the self-microemulsion delivery system of phytosterols.

\section{Abbreviations}

LDL: low density lipoprotein; POP: phytosterol oxidation product; PS:phytosterols; SMEDDS :self-microemulsifying drug delivery system ; PSSM: phytosterol self-microemulsion ; LEO:lemon essential oil; HCO-40:polyoxyethylene hydrogenated castor oil 40; PEG400:Polyethylene glycol 400; IPM: isopropyl myristate; PEG400: polyethylene glycol 400; EL: polyoxyethylene castor oil; LA: linoleic acid; OA: oleic acid; EO:ethyl oleate; PSRM :phytosterol raw materials; HPLC: high performance liquid chromatography; DL:drug loading; EE :entrapment efficiency;P :oil-water partition coefficient; $\mathrm{Km}$ : the ratio of $\mathrm{HCO}-40$ toTween $60 ; \mathrm{Kp}$ : the ratio of emulsifier to co-emulsifier; Ke: emulsifier content; PBS:phosphate buffer;TEM :transmission electron microscopy; PDI: Polydispersity index

\section{Acknowledgements}

We sincerely thank the Yuhua Guo, Yun Xu, Xue Long, Jing Jin for technical assistance.

\section{Funding}

This work was supported by the science and technology major project of the Anhui Province of China (16030701085) the open foundation of Collaborative innovation center of modern bio-manufacture, Anhui University (BM2016005).

\section{Availability of data and materials}

All data generated or analyzed during this study are included within the article.

\section{Authors' contributions}

Chuanxun Yuan and Xueru Zhang designed the study; Xueru Zhang, Risheng Jin and Peng Sun analyzed the biochemical data; Xueru Zhang and Peng Sun analyzed the Histopathological data. Chuanxun Yuan, Xueru Zhang, Risheng Jin drafted the manuscript. All authors read and approved the final manuscript. 


\section{Ethics approval}

All animals used in this study were treated strictly according to the National Institutes of Health

Guidelines of the Care and Use of Laboratory Animals. All procedures were approved by the

Animal Care Review Committee, Hefei University of technology,China.

\section{Consent for publication}

Not applicable.

\section{Competing interests}

None.

\section{References}

1. Lengyel J, Rimarčík J, Vagánek A, Fedor J, Lukeš V, Klein E. Oxidation of sterols: Energetics of $\mathrm{C}-\mathrm{H}$ and $\mathrm{O}-\mathrm{H}$ bond cleavage. Food Chemistry. 2012;133 (4) :1435-40. doi: 10. 1016/j. foodchem. 2012. 02. 031.

2. Rideout TC, Ramprasath V, Griffin JD, Browne RW, Harding SV, Jones PJ. Phytosterols protect against diet-induced hypertriglyceridemia in Syrian golden hamsters. Lipids in Health \& Disease. 2014;13(1):5.

3. Sialvera TE, Pounis GD, Koutelidakis AE, Richter DJ, Yfanti G, Kapsokefalou M, et al. Phytosterols supplementation decreases plasma small and dense LDL levels in metabolic syndrome patients on a westernized type diet. Nutrition, Metabolism and Cardiovascular Diseases. 2012;22 (10):843-8. doi: https://doi. org/10. 1016/j. numecd. 2010. 12. 004. 4. Moruisi KG, Welma 0, Opperman AM. Phytosterols/stanols lower cholesterol concentrations in familial hypercholesterolemic subjects: a systematic review with meta-analysis. Journal of the American College of Nutrition. $2006 ; 25(1): 41-8$.

5. Ribas SA, Sichieri R, Moreira ASB, Souza D0, Cabral CTF, Gianinni DT, et al. Phytosterol-enriched milk lowers LDL-cholesterol levels in Brazilian children and adolescents: Double-blind, cross-over trial. Nutrition, Metabolism and Cardiovascular Diseases. 2017;27 (11):971-7. doi: https://doi. org/10. 1016/j. numecd. 2017.09. 010.

6. Tan Z, Le K, Moghadasian M, Shahidi F. Enzymatic synthesis of phytosteryl docosahexaneates and evaluation of their anti-atherogenic effects in apo-E deficient mice. Food Chemistry. 2012;134(4):2097-104. doi: https://doi. org/10. 1016/j. foodchem. 2012. 04. 009.

7. Li Y, Li C, Li R, Chen Y, Zhang M, Guo P, et al. Associations of dietary phytosterols with blood lipid profiles and prevalence of obesity in 
Chinese adults, a cross-sectional study. Lipids in Health \& Disease. $2018 ; 17(1): 54$.

8. Kangsamaksin T, Chaithongyot S, Wootthichairangsan C, Hanchaina R, Tangshewinsirikul C, Svasti J. Lupeol and stigmasterol suppress tumor angiogenesis and inhibit cholangiocarcinoma growth in mice via downregulation of tumor necrosis factor-a. Plos One.

2017; 12 (12) : e0189628.

9. Madawala SRP, Andersson RE, Jastrebova JA, Almeida M, Dutta PC. Phytosterol and $a-1$ ipoic acid conjugates: synthesis, free radical scavenging capacity and RP-LC-MS-APCI analysis. Polish Journal of Food \& Nutrition Sciences. 2012;62(3):159-69.

10. Fu Y, Zhang Y, Hu H, Chen Y, Wang R, Li D, et al. Design and straightforward synthesis of novel galloyl phytosterols with excellent antioxidant activity. Food Chemistry. 2014;163:171-7. doi:

https://doi. org/10. 1016/j. foodchem. 2014. 04. 093.

11. Wang K, Senthil-Kumar M, Ryu C-M, Kang L, Mysore KS. Phytosterols Play a Key Role in Plant Innate Immunity against Bacterial Pathogens by Regulating Nutrient Efflux into the Apoplast. Plant Physiology. 2012;158(4):1789. doi: 10.1104/pp. 111. 189217.

12. Aldini R, Micucci M, Cevenini M, Fato R, Bergamini C, Nanni C, et al. Antiinflammatory Effect of Phytosterols in Experimental Murine Colitis Model: Prevention, Induction, Remission Study. Plos One.

2014; 9 (9) : e108112.

13. Rocha VZ, Ras RT, Gagliardi AC, Mangili LC, Trautwein EA, Santos RD. Effects of phytosterols on markers of inflammation: A systematic review and meta-analysis. Atherosclerosis. 2016;248:76-83. doi:

https://doi. org/10. 1016/j. atherosclerosis. 2016. 01. 035.

14. Calpe-Berdiel L, Escolà-Gil JC, Blanco-Vaca F. New insights into the molecular actions of plant sterols and stanols in cholesterol metabolism. Atherosclerosis. 2008;203(1):18-31. doi:

10. 1016/j. atherosclerosis. 2008. 06. 026.

15. Demonty I, Ras RT, van der Kniap HCM, Duchateau G, Meijer L, Zock PL, et al. Continuous Dose-Response Relationship of the

LDL-Cholesterol-Lowering Effect of Phytosterol Intake. JOURNAL OF

NUTRITION. 2009;139(2):271-84. doi: 10. 3945/jn. 108. 095125.

16. Botelho PB, Galasso M, Dias V, Mandrioli M, Lobato LP, Rodriguez-Estrada MT, et al. Oxidative stability of functional phytosterol-enriched dark chocolate. LWT - Food Science and Technology. 2014;55 (2) :444-51. doi: 10. 1016/j. 1wt. 2013. 09. 002.

17. Porter CJH, Trevaskis NL, Charman WN. Lipids and lipid-based formulations: optimizing the oral delivery of lipophilic drugs. Nature Reviews Drug Discovery. 2007;6(3):231-48. doi: 10. 1038/nrd2197. 
18. Pradhan S, Sagiri SS, Singh VK, Pal K, Ray SS, Pradhan DK. Palm oil-based organogels and microemulsions for delivery of antimicrobial drugs. Journal of Applied Polymer Science. 2014;131(6) :596-602.

19. Bao QY, Liu AY, Ma Y, Chen H, Hong J, Shen WB, et al. The effect of oil-water partition coefficient on the distribution and cellular uptake of liposome-encapsulated gold nanoparticles. Colloids Surf B Biointerfaces. 2016;146:475-81. doi: 10. 1016/j. colsurfb. 2016. 06. 046. PubMed PMID: 27400242.

20. Pineros I, Slowing K, Serrano DR, de Pablo E, Ballesteros MP. Analgesic and anti-inflammatory controlled-released injectable microemulsion: Pseudo-ternary phase diagrams, in vitro, ex vivo and in vivo evaluation. Eur J Pharm Sci. 2017;101:220-7. doi: 10. 1016/j. ejps. 2016. 12. 030. PubMed PMID: 28042099.

21. Xu Y, Wang Q, Feng Y, Firempong CK, Zhu Y, Omari-Siaw E, et al. Enhanced oral bioavailability of [6]-Gingerol-SMEDDS: Preparation, in vitro and in vivo evaluation. Journal of Functional Foods. 2016;27:703-10. doi: 10. 1016/j. jff. 2016. 10. 007.

22. Yoo E, Liu Y, Nwasike CA, Freeman SR, DiPaolo BC, Cordovez B, et al. Surface characterization of nanoparticles using near-field light scattering. Beilstein J Nanotechnol. 2018;9:1228-38. doi:

10. 3762/bjnano. 9. 114. PubMed PMID: 29765800; PubMed Central PMCID: PMCPMC5942383.

23. Taneja N, Singh KK. Rational design of polysorbate 80 stabilized human serum albumin nanoparticles tailored for high drug loading and entrapment of irinotecan. Int J Pharm. 2018;536(1):82-94. doi:

10. 1016/j. i jpharm. 2017. 11. 024. PubMed PMID: 29146538.

24. Sun C, Gui Y, Hu R, Chen J, Wang B, Guo Y, et al. Preparation and Pharmacokinetics Evaluation of Solid Self-Microemulsifying Drug Delivery System (S-SMEDDS) of 0sthole. AAPS PharmSciTech. 2018;19 (5) :2301-10. doi: 10. $1208 / \mathrm{s} 12249-018-1067-3$.

25. Hosseini SF, Zandi M, Rezaei M, Farahmandghavi F. Two-step method for encapsulation of oregano essential oil in chitosan nanoparticles: preparation, characterization and in vitro release study. Carbohydr Polym. 2013;95 (1):50-6. doi: 10. 1016/j. carbpo1.2013. 02. 031. PubMed PMID: 23618238.

26. Lin X, Yang S, Gou J, Zhao M, Zhang Y, Qi N, et al. A novel risperidone-loaded SAIB - PLGA mixture matrix depot with a reduced burst release: effects of solvents and PLGA on drug release behaviors in vitro/in vivo. Journal of Materials Science: Materials in Medicine. 2012;23(2):443-55. doi: 10. 1007/s10856-011-4521-2.

27. Ghosh I, Bose S, Vippagunta R, Harmon F. Nanosuspension for improving the bioavailability of a poorly soluble drug and screening of stabilizing agents to inhibit crystal growth. International Journal of Pharmaceutics. 2011;409 (1):260-8. doi: 10. 1016/j. i jpharm. 2011. 02. 051. 
28. Tian $M-P$, Song $R-X$, Wang $T$, Sun $M^{-} J$, Liu $Y$, Chen $X-G$. Inducing sustained release and improving oral bioavailability of curcumin via chitosan derivatives-coated liposomes. International Journal of Biological Macromolecules. 2018;120 (Pt A) :702-10. doi:

10. 1016/j. i jbiomac. 2018. 08. 146.

29. Wang GF, Chen QX, Huang WQ, Liu WZ, Zhang JJ. [Pharmacokinetics and relative bioavailability of ziprasidone tablets in Chinese healthy volunteers]. Nan fang yi ke da xue xue bao = Journal of Southern Medical University. 2009;29(8):1561.

30. Rutkowska E, Pajak K, Jóźwiak K. Lipophilicity--methods of determination and its role in medicinal chemistry. Acta Poloniae Pharmaceutica. 2013;70(1):3.

31. Aurijit SGEK. Hydrophobicity - Shake Flasks, Protein Folding and Drug Discovery. Current Topics in Medicinal Chemistry. 2010;10(1):-.

32. Sawant PD, Luu D, Ye R, Buchta R. Drug release from hydroethanolic gels. Effect of drug's lipophilicity (logP), polymer-drug interactions and solvent lipophilicity. Int J Pharm. 2010;396(1):45-52.

33. Dixit AR, Rajput SJ, Patel SG. Preparation and Bioavailability Assessment of SMEDDS Containing Valsartan. AAPS PharmSciTech.

2010;11 (1) :314-21. doi: 10. 1208/s12249-010-9385-0.

34. Nogueira DR, Mitjans M, Infante MR, Vinardel1 MP. Comparative sensitivity of tumor and non-tumor cell lines as a reliable approach for in vitro cytotoxicity screening of lysine-based surfactants with potential pharmaceutical applications. International Journal of Pharmaceutics. 2011;420(1):51-8. doi:

https://doi. org/10. 1016/j. i jpharm. 2011. 08. 020.

35. Jia D, Li C, Zhang Y, Yang M, Wang Y, Guo S, et al. Specific energy and surface roughness of minimum quantity lubrication grinding Ni-based alloy with mixed vegetable oil-based nanofluids. Precision Engineering. $2017 ; 50: 248-62$. doi:

https://doi. org/10. 1016/j. precisioneng. 2017. 05. 012.

36. Song D. Development of High Efficient and Low Toxic 0il Spill Dispersants based on Sorbitol Derivants Nonionic Surfactants and Glycolipid Biosurfactants. Journal of Environmental Protection. 2013;4(1):16-22. doi: 10. 4236/ jep. 2013. 41B004.

37. Pineros I, Slowing K, Serrano DR, de Pablo E, Ballesteros MP. Analgesic and anti-inflammatory controlled-released injectable microemulsion: Pseudo-ternary phase diagrams, in vitro, ex vivo and in vivo evaluation. European Journal of Pharmaceutical Sciences. 2017;101:220-7. doi: https://doi. org/10. 1016/j. ejps. 2016.12. 030.

38. Butt U, E1Shaer A, Snyder LAS, Al-Kinani AA, Le Gresley A, Alany RG. Fatty Acid Based Microemulsions to Combat Ophthalmia Neonatorum Caused by Neisseria gonorrhoeae and Staphylococcus aureus. NANOMATERIALS. 2018;8(1):51. doi: 10. 3390/nano8010051. 
39. Singh AK, Chaurasiya A, Awasthi A, Mishra G, Asati D, Khar RK, et al. Oral Bioavailability Enhancement of Exemestane from

Self-Microemulsifying Drug Delivery System (SMEDDS). Aaps Pharmscitech. $2009 ; 10(3): 906-16$.

40. Spernath A, Aserin A. Microemulsions as carriers for drugs and nutraceuticals. Adv Colloid Interface Sci. 2006;128:47-64.

41. Lipid Microemulsions For Improvin Drug Dissolution and Oral

Absorption:Physical and Biopharmaceutical Aspects. 1995.

42. Tran TH, Ramasamy T, Truong DH, Choi H-G, Yong CS, Kim J0. Preparation and Characterization of Fenofibrate-Loaded Nanostructured Lipid Carriers for Oral Bioavailability Enhancement. AAPS PharmSciTech.

2014;15 (6) :1509-15. doi: 10. 1208/s12249-014-0175-y.

43. N. S. SANTOS MAGAlHAESt HF, F. PUISIEUXS, S. BENITA" and M. SEILlERSS. An in nitro release kinetic examination and comparative evaluation between submicron emulsion and polylactic acid nanocapsules of clofibride. J MICROENCAPSULATION. 1995; 12 ( NO. 2) : 195-205.

44. Wang JJ, Sung KC, Hu OY, Yeh CH, Fang JY. Submicron lipid emulsion as a drug delivery system for nalbuphine and its prodrugs. J Control Release. 2006;115(2):140-9. doi: 10.1016/j. jconre1. 2006. 07. 023. PubMed PMID: 16959357.

45. Yong Zhang MH, Jianping Zhou, Aifeng Zou, Weize Li, 1 Chengli Yao, and Shaofei Xie. DDSolver: An Add-In Program for Modeling and Comparison of Drug Dissolution Profiles. The AAPS Journal. 2010; Vol. 12 (No. 3). doi: 10. 1208/s12248-010-9185-1).

46. Xie F, Ji S, Cheng Z. In vitro dissolution similarity factor (f2) and in vivo bioequivalence criteria, how and when do they match? Using a BCS class II drug as a simulation example. European Journal of Pharmaceutical Sciences. 2015;66:163-72. 